2017-2

\title{
Design of a Community-supported CapAble Microwave System for People with Intellectual and Physical Disabilities
}

\author{
Matteo Zallio \\ Technological University Dublin, matteo.zallio@tudublin.ie \\ Damon Berry \\ Technological University Dublin, damon.berry@tudublin.ie \\ Paula Kelly \\ paula.kelly@tudublin.ie
}

See next page for additional authors

Follow this and additional works at: https://arrow.tudublin.ie/engscheleart

Part of the Electrical and Electronics Commons

\section{Recommended Citation}

Zallio, M., Kelly P., Jakuska M., Rifai H., Berry D.,(2016). Design of a Community-Supported CapAble Microwave System for People with Intellectual and Physical Disabilities, in Springer Lecture Notes in Electrical Engineering $N^{\circ} 426$, Proceedings of Ambient Assisted Living - Italian Forum June 2016, Springer International Publishing Switzerland, ISBN: 978-3-319-54282-9 DOI: 10.1007/978-3-319-54283-6_5

This Conference Paper is brought to you for free and open access by the School of Electrical and Electronic Engineering at ARROW@TU Dublin. It has been accepted for inclusion in Conference papers by an authorized administrator of ARROW@TU Dublin. For more information, please contact arrow.admin@tudublin.ie, aisling.coyne@tudublin.ie,gerard.connolly@tudublin.ie.

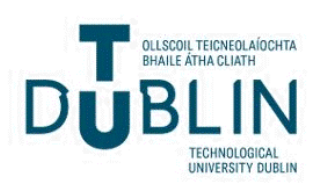




\section{Authors}

Matteo Zallio, Damon Berry, Paula Kelly, Hicham Rifai, and Modestas Jakuska 


\title{
Design of a community-supported capAble mi- crowave system for people with intellectual and physical disabilities
}

\author{
Matteo Zallio ${ }^{1}$, Paula Kelly ${ }^{2}$, Modestas Jakuska ${ }^{3}$, Hicham Rifai ${ }^{4}$, Damon Berry ${ }^{5}$
}

Corresponding Author: Dr. Matteo Zallio

Address of institution: Dublin Institute of Technology, Kevin Street Lower, Dublin 8, Ireland

Email: matteo.zallio@dit.ie

Phone Number: +353858404530

Phone Number 2: +39333 3190557

Macro-area of interest of the contribution: Well-being and active ageing, Food and nutrition.

Keywords: Design for All, ageing people, intellectual disabilities, physical impairments, smart home, capAble microwave, Community Supported Appliance, User Centred Design, Ambient Assisted Living.

\footnotetext{
${ }^{1}$ Dr. Matteo Zallio

tPOT Research group, D.I.T. - Dublin Institute of Technology, Kevin St. Lower, Dublin 8,

e-mail: matteo.zallio@dit.ie

${ }^{2}$ Paula Kelly

tPOT Research group, D.I.T. - Dublin Institute of Technology, Kevin St. Lower, Dublin 8, e-mail: paula.kelly@dit.ie

${ }^{3}$ Modestas Jakuskas

tPOT Research group, D.I.T. - Dublin Institute of Technology, Kevin St. Lower, Dublin 8,

e-mail: modestas.jakuskas@mydit.ie

${ }^{4}$ Hicham Rifai

tPOT Research group, D.I.T. - Dublin Institute of Technology, Kevin St. Lower, Dublin 8, e-mail: hicham.rifai@dit.ie

${ }^{5}$ Dr. Damon Berry

tPOT Research group, D.I.T. - Dublin Institute of Technology, Kevin St. Lower, Dublin 8, e-mail: damon.berry@dit.ie
} 


\begin{abstract}
Product personalisation has become a central topic and expected part of every day life. Different people have different characteristics and abilities and every single user has different needs which means that products have to be tailored accordingly. Microwave ovens are one of the most commonly used home appliances. People use them to quickly heat or cook certain foods. Today, according to the US Bureau of Labor Statistics, more than $90 \%$ of U.S. households own a microwave oven [11]. This project seeks to enable people with intellectual disabilities and physical impairments to perform simple cooking tasks. The target group includes ageing people who experience difficulties in using microwave systems, with a particularly low grade of autonomy in preparing foods and meals. In order to address the specific needs of the defined group of users, the research follows the principles of the Quality Function Deployment analysis and takes inspiration from the DfA (Design for All) theories and the UCD (User Centred Design) method. The aim of the research is to find new solutions in order to simplify certain activities of daily living for users who would otherwise be excluded from these actions. In the second stage, the project, seeks to provide a set of tools that enable the interaction with smart appliances to grow the sense of community, by sharing information within a social network and a Cloud-based service system. The main challenge is to create a "Community Supported Appliance" that provides technology and settings for enabling users in performing one daily activity. The CSA wants to be a simple to use, affordable learning system, continuously fed from the community of carers and service users, that has the potential, to be used, in the near future, from a wider group of people. A device of this type benefits from added value given from expert users to others from the community, in the form of sequenced multimedia instructions to the person and direct operating instructions to the appliance.
\end{abstract}

\title{
1 Introduction
}

Every single citizen has a unique set of characteristics and abilities, each user has different needs and in an era of mass-produced goods, people with physical impairments or intellectual disabilities whether those disabilities are life-long or age-related, have to live within a world that is often not tailored for them [1]. 
Design of a community-supported capAble microwave system for people with intellectual and 3 physical disabilities

Sometimes people with disabilities, are for various reasons, unable to complete what others might consider to be simple everyday tasks, such as eating, bathing, dressing, toileting, and moving - Activities of Daily Living (ADL) [2]. When people are unable to perform these activities, they need help in order to cope, either from other human beings or mechanical devices or both [3].

Innovative technologies are emerging as a support for reacting to problems related to people with disabilities and ageing people, bringing care from hospitals into the community and increasing health services into homes [4]. In particular, smart homes and smart responsive appliances address the promotion of independent living by using assistive technologies for higher quality of daily life, supporting a high degree of autonomy and dignity [5]. In these cases, the help provided by technologies for Smart Living can enhance the level of autonomy for all, they may serve as an enabling mechanism to assist the population, especially in the cognitive and security domains, and also as a mechanism that enhances their daily life [6].

Referring to the ADL activities, one of the main tasks that could enable people with disabilities and ageing people to be more independent, is related to preparing and cooking foods autonomously, by using traditional home appliances like ovens, microwaves or cookers. In order to develop a "Participatory Design Approach" a consistent group of users, from a community of physical and cognitively-disabled people based in Ireland, were involved. This was indispensable to provide feedback concerning different types of actions that users perform for eating or preparing meals.

The method used for transforming user needs into specific design inputs for designers and engineers, is the Quality Function Deployment (QFD). This method helps to create operational definitions of the specific requirements and prioritizes each product or service characteristic while simultaneously setting development targets for the product or service. This is also able to transform qualitative user demands into quantitative parameters, to deploy the functions forming quality and to deploy methods for achieving the design quality into subsystems and ultimately to specify elements for the design process [7]. By following the results, based on the continuous reverse design approach that has been conducted with the users, the research investigates how to design a userfriendly microwave system that enables people with intellectual disabilities to be more in control of their environment and to live more independent lives by participating in meal preparation.

The aim of the project is to find new solutions in order to simplify certain activities of daily living - in particular those associated with meal preparation, for users who would otherwise be excluded from these actions. This is possible by creating a working 
example of a "Community Supported Appliance" which incorporates a set of smart instruments with customised interfaces and sequenced instructions for users, in order to enable them to prepare meals independently.

The main challenge is to provide technology and a group of standard settings that give the user a chance to interact with a learning system that could be fed continuously from the user's community.

The research refers to different design approaches, combined with engineering and social sciences and a user requirements analysis borrowed from Ergonomics and Human Factors theories, in order to focus on the main issue and then to develop a prototype of the CapAble Microwave. The role of this device is to facilitate the user's task and to make sure that the user is able to use it with minimum effort [8].

\section{Cooking appliances state of art: development and use of a microwave}

King [9] defined the smart home as "A dwelling incorporating a communications network that connects the key electrical appliances and services, and allows them to be remotely controlled, monitored or accessed". A smart home is equipped with a network and smart technology such as smart lighting system, smart appliances, energy usage monitoring, security system, that enhance people's life in many aspects. Smart technologies have the potential to reduce the burden on caregivers as well as healthcare costs, while maintaining a good quality of life for its users.

Emerging smart technology can facilitate self-care, extend the self-reliance of the ageing population and disabled people, enabling them to perform different actions [10]. Focusing on one of the kitchen appliances, that is going to change radically in the next few years, the microwave is one of the most common cooking and meal heating system in use. People use microwave ovens to quickly heat prepared meals, to defrost or cook foods. Today, according to the US Bureau of Labor Statistics, more than $90 \%$ of U.S. households own a microwave oven [11].

Contemporary microwave ovens are safer, more convenient and offer a wealth of advanced features. Current models produce about $10 \%$ more power than previous versions and many include electronic sensors along with automatic controls for easy programming of cooking commands. They also come in a greater variety of sizes power ratings and styles. From small no-frills models to sophisticated ovens large enough to 
Design of a community-supported capAble microwave system for people with intellectual and 5 physical disabilities

handle full-size meals, microwave ovens are ready to meet a broad range of consumer needs.

The best news is that some of these advanced features are now available at lower prices [12]. Some vendors have produced what could be considered as the first smart microwaves, that are able to interact in a smart, productive way with the users. For example, IBM [13] introduced a concept of central coordination for different appliances for the future kitchen, while Siemens [14] produced a kitchen appliance that gathers important information from the internet (e.g. cooking recipes or cooking settings). Samsung, is one of the latest producers to release a new microwave which has a series of smart sensors, that work by reading the surface temperature of the food throughout the cooking process to decide when the food is ready, eliminating the possibility of over or under cooked food [15].

However, the evolution of microwaves from the first models produced in the early 1970 s, to the latest, integrated units with an assortment of buttons, LCD/LED screens and handle wheels, was not so linear. We can now assume that the level of easy interaction between users and microwave appliances in certain cases decreases exponentially with UI complexity. Bruner [16] assumed that categorisation processes serve to simplify the world, leading people to reserve they refined discriminatory skills only for that with which they are especially concerned. According to this theory, people usually try to simplify processes, in particular those that are repetitive, when they feel confident about using a certain device. This aspect is particularly important because it helps to understand the usability processes of different people in using the functions of various microwave ovens.

\subsection{Microwave usability analysis}

By analysing the microwave market of the last 2 years, it is possible to find a variety of different models produced by different brands. By taking a small sample of 30 of the most common microwaves starting from $€ 110$ up to $€ 340$, it is possible to analyse what kind of user interface system they have.

The analysis shows that microwave appliances have an interface ranging from a minimum of 7 buttons plus hand wheel up, to a maximum of 31 buttons. So, if we think about extrapolating the results of this investigation, to the totality of the microwaves that are available on the world's market, we can estimate that the average quantity of buttons present on a microwave is 16 (Fig. 1). 

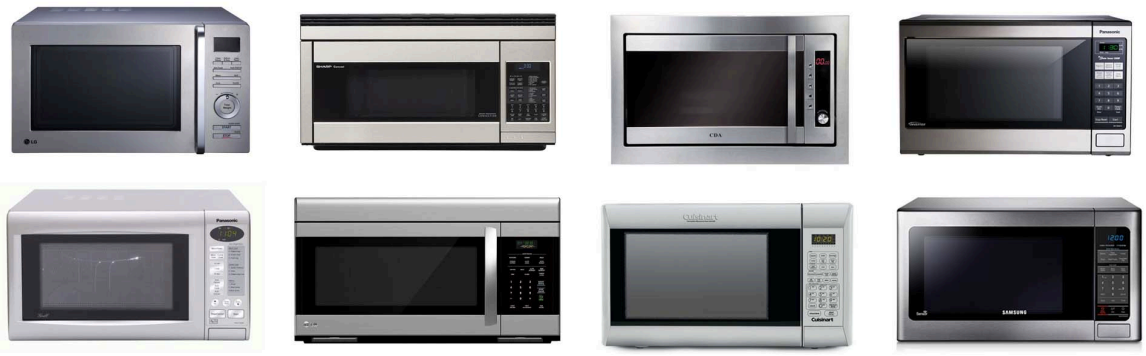

Fig. 1: Example of the most common microwaves interface which is composed by buttons and hand wheels.

By using the Quality Function Deployment method, with the users from the service community and listening to the user group experience, it was possible to notice that most of the time, members of the target group use no more than two buttons, like "add 30 sec." (which also starts the heat, in our case study) and "Stop / Cancel". If we compare the specific user needs linked to this research project and try to define an average of different users, such as ageing citizens, or people with minor physical disabilities we could say that a significant proportion of the buttons are rarely used (approximately $85 \%$ of buttons are not used).

This is caused by different aspects like: usability, complexity in interacting with the system and finally affordance. According to D. Norman an "Affordance" is the design aspect of an object which suggests how the object should be used; in particular: "...the term affordance refers to the perceived and actual properties of the thing, primarily those fundamental properties that determine just how the thing could possibly be used..." Affordances provide strong clues to the operations of things. Buttons for pushing. Knobs are for turning. Slots are for inserting things into. Balls are for throwing or bouncing. When affordances are taken advantage of, the user knows what to do just by looking: no picture, label, or instruction is needed [17].

Referring to this theory and according to the microwave analysis, based on the user's request, it has been possible to redesign the physical interface of a general microwave and set up a new interaction method that a community of primary and secondary users could use and implement continuously. 
physical disabilities

\section{Research methodology for a community-designed device}

Understanding user requirements is an integral part of information systems design and is critical to the success of interactive systems. It is now widely understood that successful systems and products begin with an understanding of the needs and requirements of the users [18]. The research, developed directly with frontline care staff and service users from the Irish-based care community, aims to identify and develop a new interface and interaction method in order to enable people with cognitive and physical disabilities to learn the cooking process and prepare meals by using a "Smart" microwave.

In the second stage, the project seeks to provide a set of tools that enable the interaction with smart appliances to grow the sense of community, by sharing information with an Internet-based instruction system. This will involve an innovative interface pattern and a set community-based instructions. Focusing on this defined group of users, it is important to underline the definition of ID - Intellectual Disability, that includes a broad range of (developing) abilities in the group of people.

Some users may demonstrate language delay, fine motor/adaptive delay, cognitive and social delay and they may, just like any other person, have difficult temperaments (differing personality traits replace for difficult temperaments), hyperactivity, disordered sleep and associated behaviours may include aggression, self-injury, defiance, inattention, hyperactivity, sleep disturbances [19].

\subsection{Quality Function Deployment method for a User Centred Design approach}

The Quality Function Deployment is a team user-based analysis that provides a way for identifying and transferring user requirements into technical specifications for designing devices, processes and products. The term Quality Function Deployment derive from a Japanese translation of this methodology, hin shitsu (quality), ki nou (function), ten kai (deployment). The methodology consists of a structured procedure that starts with the qualities desired by the users, leads through the functions required to provide these products and services and identifies the means for deploying the available resources to best provide, in this particular case, the new microwave interface [20]. 
In order to address the specific needs of the group of users, while following a path which provides a usable and affordable product for ID users, the research takes inspiration from merging the principle dictated from the Design for All theories and the User Centred Design method. The Design for All is the intervention into environments, products and services, which aims to ensure that anyone, including future generations, regardless of age, gender, capacities or cultural background, can participate in social, economic, cultural and leisure activities with equal opportunities [21].

The User Centred Design approach originated in Donald Norman's research laboratory at the University of California San Diego (UCSD) in the 1980s and became widely used after the publication of a co-authored book [22]. D. Norman, offers four basic suggestions on how a design should be:

- Make it easy to determine what actions are possible at any moment.

- Make things visible, including the conceptual model of the system, the alternative actions, and the results of actions.

- Make it easy to evaluate the current state of the system.

- Follow natural mappings between intentions and the required actions, between actions and the resulting effect and between the information that is visible and the interpretation of the system state [23].

By following the QFD method, the project team employed frequent meetings (one every two weeks for four months) with service users and front line care staff. The meetings were indispensable to gather feedback on the developing system from primary users, a significant number of the 40 residents at the community site, in addition to feedback and indications from secondary users (in particular the caregivers and allied health professionals who provide services inside the community).

Different instruments were devised in order to collect the feedback and the most useful and productive were: a customized online survey, which was administered by the community caregivers, direct interviews with the service users, which occurred during the team meetings with the support of the caregivers and finally a focus group with different persons. The study group was comprised of interviewees and focus group members from one location; overall 10 suitable individuals took part in the interviews and focus groups out of a community of 40 service users.

A wider community of survey participants was approached across four locations reaching a group of approximately 120 people. One of the groups of interviewees and focus group was made up of 4 individuals ranging in age from 27 to 47 years old. They 
Design of a community-supported capAble microwave system for people with intellectual and 9 physical disabilities

were identified as they all had differing individual support needs in order to use the microwave however, limited literacy skills affect the 4 participants to varying degrees.

The specifics abilities of those four people are related to:

- Limited literacy to be defined in this instance but not limited to the ability to understand and recognize some words in different contexts, for example on a door push/pull and road signs such as Stop.

- The ability to read /write their own name, but unable to write freely from instruction or memory.

- Recognizing brand names/commonly understood items with support of logos and other environmental clues.

The interviews were conducted on a one-to-one basis in an office environment and were carried out by an experienced and familiar care staff to the interviewees and they were asked about their experiences in using microwaves. The objective of the interview was to:

- Identify positive and negative interactions while using microwaves.

- The possible reasons and barriers that shaped the interactions.

- User patterns/trends, what and when people cook.

All of this information would help in providing data for shaping the "Persona" model in the following stage of research. The second, more detailed interviews took place with the same interviewers in a kitchen which was familiar to the interviewees using two different microwaves. The first microwave has two wheels, one for time and one for setting. The second microwave has a digital screen with a number keypad and pre-set functions. In order to capture useful information on the interaction between person and microwave, users were asked to perform four basic tasks:

- Open door/close door.

- Set up the timer for 2 minutes or 5 minutes.

- Identify full/half/defrost functions.

- Highlight any dangers while using microwave 
Information was gathered while observing the interactions between each single user and the device. By referring to a reverse process, information was also used to shape the questions and statements in the following focus group. After the initial interviews it was possible to move towards focus groups, which was composed of 6 additional individuals who joined the first 4 users. The objective of the focus group was to ask a wider cross section of user's opinions on the interaction and usage of the microwave. The focus group was held in an office environment, with the presence of the microwaves and 6 additional members; 3 of them were males and 3 females ranged in age from 30 to 55 years old, with similar abilities to the people belonging to the first group of interviewees.

The interview process was the same as the previous one, asking questions related to the usage of the microwave and observing interactions to identify any common problems which were not verbally communicated, in order to collect as much feedback as possible to build the Persona model. Finally, support staff joined in at the end of the focus group to help provide insight from their perspective, as they usually provide daily support to the focus group while using the microwave. It emerged from the focus group that users, especially an individual user with cognitive disabilities, like to watch cartoons on the TV and so they prefer to look at images, or sketches, rather then reading text or numbers (this is also due to a lack of literacy). Some of the users also like to interact with apps and games on smartphones or tablets, because those devices provide direct feedback and enrol the user in participating in an interactive learning process for increasing abilities. From initial interviews and focus group an accessible survey was developed in order to increase the case study size. This was made available across 4 different locations, reaching a group of approximately 120 persons and it was designed to be accessible for people with limited literacy. It has been possible to receive $30 \mathrm{com}$ pleted surveys, which defined the main user requirement analysis. (Fig. 2).

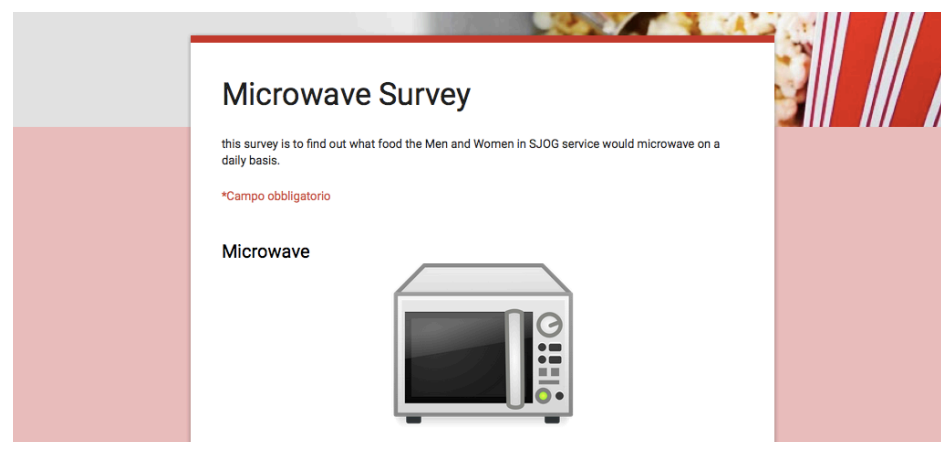

Fig. 2: Extract of the online survey. What food the users would microwave on a daily basis? 
Design of a community-supported capAble microwave system for people with intellectual and physical disabilities

Generally, it has been possible to collect feedback related to the request of support for using the microwave and more than the $66 \%$ of people responses were positive (Fig. $3)$.

\section{Do you need support to use the microwave?}

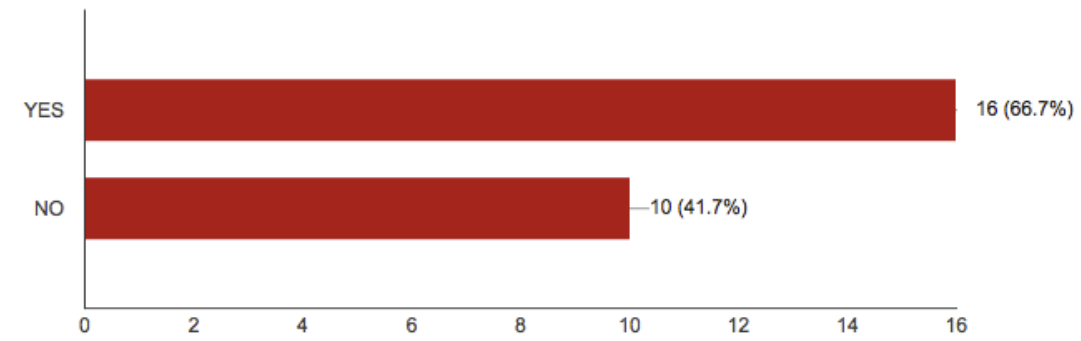

Fig. 3: Online survey for primary users. Do you need support to use the microwave?

For ID users, as for older people, the safe use and correct operation of a microwave can be a big challenge, mainly caused by interface usability issues. Settings (product to cook or heat, time and temperature), the often-heavy mechanical catch/interlock on microwave doors, the multi-stage process that they have to complete in order to prepare a good quality meal, are some of the main issues identified during the interview, focus groups and online survey.

In these circumstances and for these users, a modified and improved microwave represents an opportunity to be more autonomous, to feel empowered to use technology which was formerly unusable for them and the increased independence of providing hot food by themselves.

\subsection{Designing with the "Persona" model}

The set of needs collected within the interviews, focus groups and survey, were shaped by using the "User Persona" model. A Persona is a model of a user that focuses on the individual's goals when using an artifact. The model has a specific purpose as a tool for software and product design. The Persona resembles classical user profiles, but with some important distinctions. It is an archetypical representation of real or potential users and it is not a description of a real, single user or an average user. 
The Persona represents patterns of user's behaviour, goals and motives, compiled in a fictional description of a single individual. It also contains made-up personal details, in order to make the Persona more "tangible and alive" for the development team [24].

Thanks to this fundamental instrument, it has been possible to summarize the way that people interact with a microwave and what they usually cook for lunch or for dinner in the common microwave room. This was a key topic, because as every experienced microwave user, knows different foods, have different heating temperatures and times. In addition, for some foods, like for example soups, popcorn and noodles, the user has to work through a multistage process. They will not just put the prepared meal into the microwave, and switch it on, but they may also need to fill a vessel up with water, preheat an item and stir, then heat again, or for example shake the popcorn box, before and/or in the middle of the cooking process. In addition to the power setting of the microwave itself, food entering the microwave could come from a fridge, freezer or cupboard, necessitating slightly different cooking times.

Finally, it is important to underline that when a food is overcooked there is a big danger of burns when handling the pot or the food container for this target group. In order to avoid this issue, a humidity sensor that is based on the generation of moisture vapour from the food, could shuts off the microwave when a certain level of humidity is reached inside the cavity and a temperature sensor could be also used to determine when the meal is cooked and safe to handle.

For this work three Persona models have been created to represent the goals and behaviour of the group of users and synthesized from data collected from interviews with the users.

James, Persona 1, is a male aged 29. He is extremely independent, he can travel freely in the local community and he is adept in the use of technology such as IPad and IPhone. There are no physical challenges that would affect James in using a microwave but unfortunately he has limited literacy skills and finds difficult to use numbers in day to day life. This aspect has been previously identified during phone training where alternatives for an unlocking pin were required due to his multiple wrong attempts blocking the use of the phone. Finally James cannot differentiate between the numbers and setting of the microwave timer correctly, so it is important to solve this issue in order to enable him to use the microwave correctly.

Christine, Persona 2 , is a female aged 45 . She would require extra supports to complete activities of daily living. She uses a motorized wheelchair and has some fine motor skill limitation with hand grip and arm reach across both sides of her body. She also has limited literacy and basic numeracy skills. The identified support needed for Christine is the physical use of the microwave. As the accuracy of touch is decreased at full 
Design of a community-supported capAble microwave system for people with intellectual and physical disabilities

stretch, she may press the wrong number by accident not by misunderstanding. She also needs a system that enables the opening of the microwave door with one hand.

John, Persona 3 is a male aged 37 . He is highly functioning and possess the majority of skills for independent participation in activities of daily living. He can freely access local areas and has excellent independent skills. He has good numeracy skills that enable him to easily manage money and successfully use the facilities of a bank/ATM. John has the best literacy level of the group and he can recognize most words in context and can understand or decipher information presented in written form. He uses hearing aids and has some sight loss which can affect reading small printed text. The main support that is needed for John is to understand the effect of the actions/settings on a particular device. It is likely that John set the numbers correctly each time, with the proper sized font, but he might find issue with the functions of the microwave, for example if he cooked the food for the correct time but on the wrong setting.

The results from the interview with primary users and consultations with caregivers allowed the creation of three different representations of the group of users. It is also important to underline that results from the survey (more than $58 \%$ of people need support for preparing food packaging, e.g. peel of lid) (Fig. 4) and the main issues related to poor literacy of Persona 1 and 2 are very important to understand that not only the cooking process has to be automated by the microwave, but there should be some aid for the process of preparing the food packaging.

\section{Do you need support with the instructions on how to prepare the packaging? For example peel of the lid.}

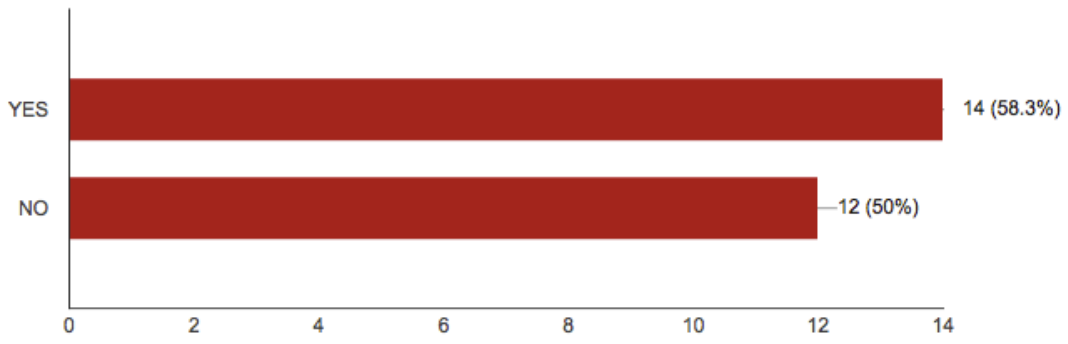

Fig. 4: Online survey for primary users. Do you need support with the instructions on how to prepare the packaging? For example, peel of the lid. 
In light of this information, the research group focused on defining a common and easy way to use a low cost technology that enables people with intellectual disabilities to use the microwave, but also one that could (according to Design for All and User Centred Design) be appreciated by the caregivers and family members as users.

\subsection{Preferences from the users}

One of the most important elements that was highlighted by the survey, was that people from the care community, are accustomed to go to the supermarkets with the caregivers, where they are given the opportunities to feel independent by choosing prepared meals from the shelves and scanning products at the self-cashier. This is an enormous challenge for those people who can, followed by a caregiver, choose and buy foods without any particular help, by using the facilities provided by the supermarkets (automated barcode readers and automatic cashiers).

The results of the survey indicate that more than half of the users use the microwave under supervision, to reheat a meal, that was previously cooked (like prepared meals from supermarkets which have a big portion for just one person) and that $25 \%$ use the microwave for cooking a packaged microwave meal (Fig. 5).

\section{What are you using the microwave to do?}

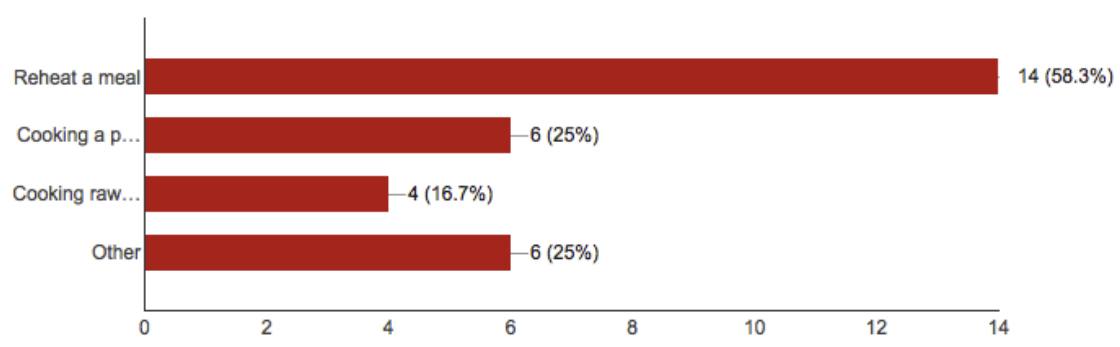

Fig. 5: Online survey for primary users. What are you using the microwave to do?

These indications brought to light that the majority of this community of users employ a microwave to cook or heat prepared or semi-prepared food, with the help of caregivers. So for the purposes of the work it was possible for the project team to narrow down the field of action of smart system linked to the microwave.

Packaged and processed food from supermarkets has some drawbacks, but also has the advantage of extensive labelling. Apart from the printed label, the barcode provides 
Design of a community-supported capAble microwave system for people with intellectual and physical disabilities important and distinctive information which allow users and (in this case) a capAble microwave, to understand with simply one action, which kind of food is contained in the packaging and provide the possibility to initialise the microwave in the best way. A barcode is a machine-readable arrangement of numbers and parallel lines of different widths printed on a package, which can be electronically scanned at a checkout to register the price of the goods and to activate computer stock-checking and reordering also called Universal Product Code, UPC [25].

Barcodes, have a huge potential as an identifier of data related to different products and provide an opportunity to design different pre-sets, for various types of food.

\section{The capAble microwave}

The outcomes of the user analysis, allow us to identify the principles that have driven this smart innovation.

People with disabilities and some ageing people with particular medical conditions have difficulties in recalling very simple sequences of actions. The new microwave device seeks to provide not just cooking assistance, but also a support for independent living, by interacting in a productive way with the user.

As mentioned in the user feedback analysis, some of the users like to interact with apps, games, watching animated movies with the aid of different devices and enjoy scanning products in the supermarkets. One of the key points of the interaction process, could be researched is the "gamification" of the cooking process. In recent years, gamification, the use of game design elements in non-game contexts, has seen rapid adoption in the software industry, as well as a growing body of research on its uses and effects [26].

In order to create a smart system by using not only hardware technologies, but also user friendly software, it has been important to design an interface that allows people to easily understand how to use hardware, that evokes emotions, feelings and a sort of challenge by performing a particular process. This process is known as gamification and users and caregivers, from the community were conscious of the potential of this interaction instrument. So, by following these indications, it has been possible to set the hardware structure, which is composed of a barcode scanner, a touchscreen, an audio speaker a Wi-Fi module and a Raspberry PI, but also understand how to represent the visual feedback on the screen in a communicative way. 
One of the simplest and more communicative interfaces, for the service users in the care community that they usually like to see and to interact with, are "animations"; simple animations were introduced in the project, to make information accessible in the community. The regular use of Adobe voice (an application for a tablet, which allows for the creation of easy and professional looking animations), gives service users experience with interacting with information in this format.

In other previous experiences, caregivers had positive feedback from using anonymous animated instruction sequences, or simple and clear sketches and drawings in order to communicate some desired interaction to the users. So it was decided, with the help of the caregivers, to move forward on developing a simple and easy "animation based" interface, which could be detailed, but at the same time that would give a clear idea of the sequenced actions that everyone has to perform for cooking a particular meal. The caregivers and service users of the community collaborate to create multimedia instructions for the end-users and operating instructions for the associated appliance. These instructions can be managed "in the Cloud" in what we term the instruction management service.

When a user scans a barcode, the barcode information, and information about the device that is requesting instructions are sent to the instruction management service, which responds by sending customised cooking instructions to the requesting device. The device acts in the manner of a browser, by issuing multimedia instructions to the user, prompting them to engage in the cooking process. At certain points in the cooking process, the device also operates the controls of the microwave to cook the product.

By enabling users to scan the barcode of foods close to the microwave, the device "understands" what kind of food it is, identifies the appropriate cooking time and temperature and provides visual and audio feedback to the user.

\subsection{System architecture of the capAble microwave}

The capAble microwave has the potential to give different types of feedback to the user, before starting the automatic cooking process. Two embedded functions are at the core of the interaction system:

1. Suggestion: by pointing out a set of instructions in order to guide the user in the cooking/heating process, the system is able to give instructions not only in directly controlling the appliance presets for temperature, time, but also about what the user could do in order to prepare the food package to be heated. Actions like: peel off the

film, add some water, stir the food, shake the bag (for popcorn), etc. can be promoted 
Design of a community-supported capAble microwave system for people with intellectual and

physical disabilities

by the microwave touchscreen interface by enabling user interaction and by making the cooking process more interactive, adaptable and that can be turned into a game, rather than a automatic, boring process.

2. Alerts: it is known that the heating/cooking process has safety concerns, such as e.g. the risk of fire, if someone forgets to turn off the microwave, or the risk of heating the meal too much, or the risk of the food "going off" if the food is left inside the microwave for too long a period. The timer and temperature alerts, settled with the specific information given by the barcode and on-line services, could be linked to the Cloud network of the community or to the smartphone/tablet of the carer or end user, providing a safer control of the process while avoiding personal security risks.

In order to solve the different user issues, the microwave uses information that are downloaded from the Cloud. Members of the community can upload different user instructions and settings for different types of microwaveable food products to the platform. The platform created on the "easycook.ie" domain (Fig. 6), is a web-service, where people from the community can upload information related to each food (like temperature and cooking time, calories, nutritional information, etc.), bought in supermarkets and simultaneously each microwave of the community can get information thanks to this instruction repository.



Fig. 6: Prototype of the "easycook.ie" website for the instruction repository.

This web-based service could be seen, not only as a basic information service in the initial stage of the research, but also as a learning tool that enables people to upload additional information and grow the sense of community for different group of users. The capAble microwave effectively becomes an interactive smart object, which provides the required food information and gives help in following the cooking process. So, by interacting with users and redesigning the electronic circuit, the research moves forward 
on producing a smart add-on module for existing microwaves such as those used by the community of care. A barcode scanner with a Raspberry Pi, a small touchscreen that gives visual feedback a speaker that provides audio feedback and a Wi-Fi module are the core elements that comprise the first prototype (Fig. 7).

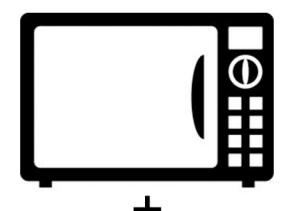

$+$

INTERACTION OBJECTS

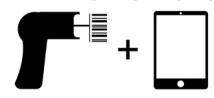

$\downarrow$
EMBEDDED

\section{CLIENT}

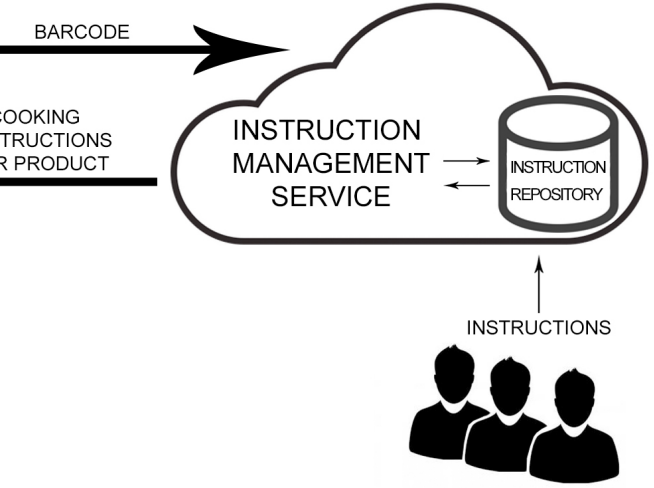

COMMUNITY OF SERVICE USERS AND CARERS

Fig. 7: Architecture diagram of the capAble microwave prototype.

Another important positive issue that moves on developing the easily accessible, "animation based" interface, is to "keep it simple" and "not personal" (so for example, without showing a particular bowl, taken from just a photo of one user's bowl) it avoids a confusion that occurs when a service user sees a photo of a real bowl. In that case, there was an experience in one of the interviews, that one user could say "it's not my bowl, it's Jack's bowl" and this could be a negative point that could interrupt the process of good interaction between ID user and the smart microwave.

The capAble microwave will be enabled to inform the user what kind of food is contained inside the packet just scanned and it will provide assistance for supporting with the instructions on how to prepare the packaging and with the safe opening of the microwave door. By recognising the type of food scanned, which is linked to a set of information (like cooking time, temperature, microwave power) provided on the easycook.ie website, the microwave will become an interactive device that has the capacity to remind users of the different tasks.

For example: peel off the film, or add some water and then when the user places the product inside the microwave it will automatically start the appropriate cooking/heating process. Basically, by using an integrated barcode scanner, a Wi-Fi module, a touchscreen, added to the existing microwave unit and by developing the interaction 
Design of a community-supported capAble microwave system for people with intellectual and physical disabilities software, it is possible to transform a classical, difficult-to-use microwave, into an instrument that enables user engagement and that also simplifies the process for varying groups of users to the extent that the required number of buttons is ideally reduced to one.

\section{Conclusion}

The aim of the project is to find new solutions in order to simplify certain activities of daily living for users who would otherwise be excluded from these actions. This is possible by creating a working prototype of a "Community Supported Appliance" which incorporates a set of tools that a community of primary users and secondary users could use and implement, in order to address the main challenge of the cooking process.

By working on the hardware and software technologies and on the interface of the new microwave appliance, it has been possible to share cooking information and to enable people with intellectual and/or physical disabilities to learn from their actions, adding value between members of the community.

The CSA - Community Supported Appliance has the potential to become an instrument that enables people to perform actions and to re-design customized interfaces for different home appliances. In perspective, the CSA, can be applied within different scenarios, like the home environment or within the public spaces services or private/ public transportation context.

The main challenge is to provide the technology and settings that give the possibility to have a learning system that could be fed continuously from the user community of carers and service users. The actual potential of this smart community-based system could be much more significant, if we consider the millions of users that usually operate microwaves just to heat or cook meals. We are conscious that the usage of a barcode scanner could have limits, related to specific foods, but the feedback derived from the first tests with the users group, has shown that the microwave appliance is mostly used not for the "proper cooking" of a meal, but just for heating or preparing "ready-made" foods. So by introducing this small add-on system to a microwave, we could significantly increase the possibility of enabling people with disabilities to independently prepare foods, but at the same time give to everyone a more convenient way to interact with a microwave. 
As previously noted, although the focus group in this case was a community of people with a range of intellectual and physical disabilities, this solution should not be limited only to this cohort. Clearly this type of solution can be applicable to a range of possible user groups. This system could also allow users with other abilities (like ageing people and children) to prepare a safe heated product and finally to save time by reducing the number of actions and the cognitive load that is required to cook/heat a meal in the best way.

Data shows that in 2002 in US, the health related expenditures was more than $\$ 61 \mathrm{~B}$ [27] and the health and caregiver system will be increasingly stressed as those numbers increase. Chronic diseases like Dementia, Alzheimer's Disease and a variety of physical impairments can make ADL very difficult.

For some older citizens, even the simplest of tasks such as cooking a microwaveable meal can become overwhelming due to the complex cooking instructions written in small print on the cooking package and the need for memory and coordination in interacting with the microwave oven [28]. So there is a substantial and growing need to design new interactive systems, based on known actions and known human behaviour, in order to keep people as independent as possible in their environment. This will also affect the process of care provision and the community welfare structure, which will take advantage of these solutions, by reducing the costs and effort associated with private assistance [29]. This research seeks to define a solution related to a particular problem within a community care context and give a suggestion to apply the idea in parallel contexts. Assisted homes and retirement homes users, could help in a further develop of the capable microwave that could become a Universally Designed and functional smart appliance for everyone's house.

This prototype could become an important and useful case study that should be further investigated and integrated in a range of new cooking appliances, by worldwide home appliance producers.

New microwaves, like the Samsung previously mentioned, with additional sensors, will add potential to have smarter community supported cooking appliances with improved safeguards such as warnings to end-users about food temperature. This smart development, apart from the community-support aspect (which is not so developed in the automotive sector) is nevertheless quite similar to the evolution that has already occurred in cars, with new sensors and smart systems. 
Design of a community-supported capAble microwave system for people with intellectual and

physical disabilities

\section{References}

1. Howe M. J. A., Principles of Abilities And Human Learning, East Sussex: Psychology Press, 1998.

2. Wiener J. M., Raymond J. H., Clarck R., Van Nostrand J. F., Measuring the Activities of Daily Living: Comparisons Across National Surveys. US Department of Health and Human Services, Journal of Gerontology: Social Sciences, Vol. 45, No. 6, pp. S229-237, 1990.

3. Fillenbaum, Gerda G., Activities of Daily Living. In George L. Maddox (Ed.), The Encyclopedia of Aging. New York: Springer, 1987.

4. Taylor K., Connected health. How digital technology is transforming health and social care. The Creative Studio at Deloitte, London, 2015.

5. D'Ulizia A., Ferri F., Grifoni P., Guzzo T., Smart Homes to Support Elderly People: Innovative Technologies and Social Impacts, in "Pervasive and Smart Technologies for Healthcare: Ubiquitous Methodologies and Tools", Medical Information Science Reference; First edition, Hershey, PA, 2010.

6. R.W. Pew, S. B. Van Hemel, "Technology for adaptive aging", Steering Committee for the Workshop on Technology for Adaptive Aging, National Research Council of The National Academies, p. 247, 2004.

7. Yoji A., Development History of Quality Function Deployment. The Customer Driven Approach to Quality Planning and Deployment. Minato, Tokyo 107 Japan: Asian Productivity Organization. 1994.

8. D. Norman, "The design of everyday things". New York: Doubleday, 1988.

9. King, N. (2003) Smart Home - A Definition, Housing LIN Intro Factsheet, 2003.

10. Chernbumroong S., Atkins S. A., Yu H., Perception of Smart Home Technologies to Assist Elderly People, in Proceedings "The 4th International Conference on Software, Knowledge, Information Management and Applications" (SKIMA 2010), Paro, Bhutan, 2010.

11. Source, Cox and Alm, "Time Well Spent: The Declining Real Cost of Living in America", in Federal Reserve Bank of Dallas - 1997 Annual Report; see Exhibit 8 on page 22, 1997. http://www.dallasfed.org/htm/pubs/pdfs/anreport/arpt97.pdf. Cited 10 March 2016.

12. P. R. Liegey Jr., "Hedonic Quality Adjustment Methods for Microwave Ovens in the U.S. CPI." 2001. Document from: http://www.bls.gov/cpi/cpimwo. Cited 15 March 2016.

13. Source: IBM Kitchen Demo. http://www.ngi.ibm.com/demos/kitchen.html. Cited 18 February 2016.

14. Source: SIEMENS Synco living Website: http://www.siemens.com/about/sustainability/en/environmental-portfolio/products-solutions/building-technology/home-automation.htm. Cited 19 February 2016

15. Source: Samsung "Smart Oven" http://www.which.co.uk/news/2013/10/which-tries-samsungssmart-microwave-oven-335016/ Cited 19 February 2016.

16. Bruner J. S., On perceptual readiness. In Psycologycal Review, Vol. 64, pg. 123,152, 1957.

17. D. Norman, "The design of everyday things". New York: Doubleday, page 9, 1988.

18. Maguire M., Bevan N., User requirements analysis. A review of supporting methods, in Proceedings of IFIP 17th World Computer Congress, Montreal, Canada, 25-30 August 2002, p133-148. Kluwer Academic Publishers, 2002.

19. A. S. Zeldin, A. T. F. Bazzano, 2014, "Intellectual disability", paper in Medscape, source: http://emedicine.medscape.com/article/1180709-overview. Cited 24 February 2016. 
20. Guinta, L. R., Praizler, N. C., The QFD Book, The Team Approach to Solving Problems and Satisfying Customers Through Quality Function Deployment. AMACOM Books. 1993.

21. Citation from: http://designforall.org/design.php. Cited 12 March 2016.

22. A. Chadia, D. Maloney-Krichmar, J. Preece. "User-centered design." Bainbridge, W. Encyclopedia of Human-Computer Interaction. Thousand Oaks: Sage Publications, pages 445-456, 2004.

23. D. Norman, "The design of everyday things". New York: Doubleday, page 188, 1988.

24. S. Blomkvist, Persona - an overview, Extract from the paper "The User as a personality. Using Personas as a tool for design", Position paper for the course workshop "Theoretical perspectives in Human-Computer Interaction" at IPLab, KTH, September 3, 2002.

25. Definition from Collins English Dictionary - Complete \& Unabridged 2012 Digital Edition William Collins Sons \& Co. Ltd. 1979. Cited 22 March 2016.

26. S. Deterding, S. L. Björk, L. E Nacke, D. Dixon, E. Lawley, "Designing Gamification: Creating Gameful and Playful Experiences", Paper in "CHI'13 Extended Abstracts on Human Factors in Computing Systems", ACM Publisher, pages: 3263-3266, 2013.

27. R. Koppel, "Alzheimer Disease: The Cost to U.S Business in 2002", Source: http://www .alz.org/Media/newsreleases/2002/062602ADCosts.pdf . Cited 12 March 2016.

28. J. Russo, A. Sukojo, S. Helal, R. Davenport, and W. Mann, "SmartWave Intelligent Meal Preparation System to Help Older People Live Independently," Proceedings of the Second International Conference on Smart homes and health Telematic (ICOST 2004), Singapore, September 2004.

29. Zallio M., Home automation and technology: renewal of existing buildings, in Spadolini M. B., Design for better life. Longevità, scenari e strategie, pp. 102- 110, Franco Angeli, Milano, 2013. 